\title{
GUIDELINES
}

\section{Diagnosis and treatment of prostate cancer: summary of NICE guidance}

\author{
John Graham, ${ }^{1}$ Mark Baker, ${ }^{2}$ Fergus Macbeth, ${ }^{3}$ Victoria Titshall, ${ }^{3}$ on behalf of the Guideline \\ Development Group
}

${ }^{1}$ Taunton \& Somerset NHS Foundation Trust, Musgrove Park Hospital, Taunton TA1 5DA

${ }^{2}$ Leeds Teaching Hospitals NHS Trust, St James's University Hospital, Leeds LS9 7TF

${ }^{3}$ National Collaborating Centre for Cancer, Cardiff CF10 3AF

Correspondence to: J Graham john.graham@nhs.net

BMJ 2008;336:610-2 doi:10.1136/bmi.39498.525706.AD
This is one of a series of $B M J$ summaries of new guidelines, which are based on the best available evidence; they will highlight important

recommendations for clinical practice, especially where

uncertainty or controversy exists.

The supporting evidence

statements and further

information about the guidance

are in the version on bmj.com.

\section{Why read this summary?}

Prostate cancer is one of the commonest cancers in men. Each year there are about 35000 new cases in England and Wales and over 9000 deaths. The clinical course can extend over many years and often involves many different healthcare professionals. Evidence exists of practice variation around the country and of patchy availability of certain treatments and procedures. This article highlights a selection of the most recent recommendations from the National Institute for Health and Clinical Excellence (NICE) on the diagnosis and treatment of prostate cancer. ${ }^{1}$

\section{Recommendations}

NICE recommendations are based on systematic reviews of the best available evidence. When minimal evidence is available, recommendations are based on the guideline development group's opinion of what constitutes good practice. Evidence levels for the recommendations are in the longer version of this article on bmj.com.

\section{Informed decision making}

Men with prostate cancer can be faced with multiple treatment choices over many years. Healthcare professionals should:

- Adequately inform men with prostate cancer and their partners or carers about the effects of prostate cancer and the treatment options on their sexual function, physical appearance, continence, and other aspects of masculinity.

- Support men and their partners or carers in making treatment decisions, taking into account the effects on quality of life as well as survival.

- Ensure that mechanisms are in place to allow men with prostate cancer and their primary care providers to gain access to specialist services throughout the course of their disease.

\section{Diagnosis}

- To help men decide whether to have a prostate biopsy, discuss with them their prostate specific antigen (PSA) level, digital rectal examination findings, age, black African or black Caribbean ethnicity (associated with an increased risk of prostate cancer), and comorbidities (which may affect your decision to offer curative treatment), together with any history of a previous negative prostate biopsy. The serum PSA level alone is a poor predictor of the presence of prostate cancer and should not automatically lead to a prostate biopsy; moreover, many cancers diagnosed on this basis alone will be of low risk, causing little or no impact on life expectancy.

- The results of all prostate biopsies should be reviewed by a urological cancer multidisciplinary team. Men should only have a repeat biopsy after a review by this team of the risk characteristics, including life expectancy, PSA level, digital rectal examination, and prostate volume.

\section{Localised disease}

- Urological cancer multidisciplinary teams should assign a risk category (table) to all men with newly diagnosed localised prostate cancer.

One of the greatest challenges in prostate cancer is deciding which men have rapidly growing cancers that need treating and which men have indolent cancers that will never trouble them.

- Given the range of treatments and their serious side effects, men with prostate cancer who are candidates for radical treatment should have the opportunity to discuss their treatment options with a specialist surgical oncologist and a specialist clinical oncologist.

- Men having radical external beam radiotherapy for localised prostate cancer should receive a minimum dose of $74 \mathrm{~Gy}$ to the prostate at no more than 2 Gy per fraction.

- The new techniques of cryotherapy and high intensity focused ultrasound are not recommended outside clinical trials as further evidence of their long term clinical effectiveness and safety is required. 
Low risk

- For men with low risk, localised prostate cancer (table) who are considered suitable for radical treatment, first offer active surveillance (measurement of PSA level and repeat biopsies). Such surveillance is recommended (rather than immediate radical treatment) because of the low mortality in men with marginally raised PSA levels, but for a small proportion of men with low risk disease, surveillance may not be appropriate. Changes in PSA level and Gleason grade are important prognostic factors and may identify cancers that require radical treatment.

- For men with localised prostate cancer who have chosen an active surveillance regimen and who have evidence of disease progression (that is, a rise in PSA level or adverse findings on biopsy), offer radical treatment.

Thus radical treatment is offered in active surveillance but deferred until there is evidence of progression of the cancer as defined by changes in PSA levels or Gleason histological grading on repeat biopsy.

\section{Intermediate risk}

Intermediate risk, localised prostate cancer can be treated with radical surgery, radical radiotherapy, or active surveillance.

High risk

- Active surveillance is not recommended for men with high risk, localised prostate cancer.

- Offer radical prostatectomy or external beam radiotherapy to men with high risk, localised prostate cancer when there is a realistic prospect of long term disease control.

Relapse after radical treatment

- Biochemical relapse (a rising PSA level) alone should not necessarily prompt an immediate change in treatment but should trigger serial measurements of PSA to calculate the PSA doubling time.

- For men with biochemical relapse after radical prostatectomy who have no known metastases, offer early radical radiotherapy to the prostate bed.

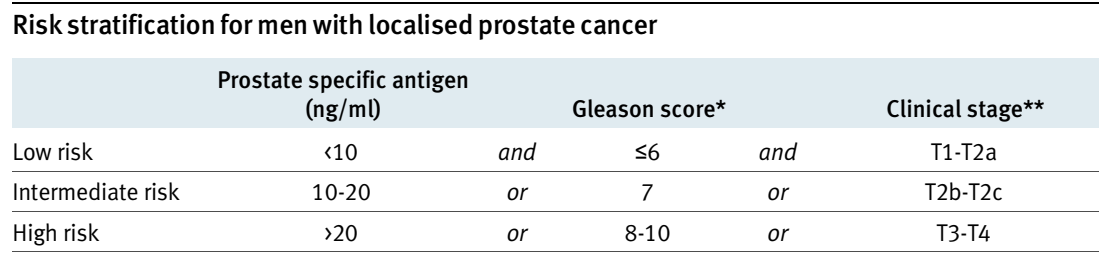

*The sum of the predominant histological pattern of cancer (graded from 1 to 5 ) and the next most common pattern. For biopsies (as opposed to radical prostatectomy specimens) it is not possible to allocate a pattern of $<3$ because of the small quantity of tissue obtained. Therefore the lowest possible Gleason score on a biopsy is $6(3+3)$.

**The anatomical extent of the cancer, informed by the gross resection specimen (in men having prostatectomy) or by biopsy and rectal findings, sometimes augmented by magnetic resonance imaging. T1-T2a describe low volume disease confined to $<50 \%$ of one prostatic lobe. T3 and T4 cancers extend beyond the prostate.
- Hormonal therapy is not routinely recommended for men with biochemical relapse unless they also have symptomatic local disease progression or any proved metastases or a PSA level that has doubled in less than three months.

\section{Hormonal therapy}

- Offer bilateral orchidectomy to all men with metastatic prostate cancer as an alternative to continuous luteinising hormone-releasing hormone agonist therapy (gonadorelin analogue therapy).

- Offer intermittent androgen withdrawal with the caveat that there is no long term evidence of its effectiveness but it may reduce side effects such as hot flushes.

\section{Hormone refractory disease and palliative care}

- When men with prostate cancer develop biochemical evidence of disease refractory to hormone therapy, their treatment options should be discussed by the urological cancer multidisciplinary team with a view to seeking an oncological and/or specialist palliative care opinion as appropriate.

- For men with metastatic prostate cancer, offer tailored information and access to specialist urology and palliative care teams so that their specific needs can be tackled. They should have the opportunity to discuss any important changes in their disease status or symptoms as these occur.

- Evidence exists that chemotherapy can prolong life and improve symptoms but that it should be given before the man's performance status (general wellbeing) deteriorates. Docetaxel chemotherapy is recommended, within its licensed indications, as a treatment option for men with hormone refractory metastatic prostate cancer only if their Karnofsky performance status score is $60 \%$ or more (requiring occasional assistance, but able to care for most personal needs).

\section{Bone metastases}

- The use of bisphosphonates to prevent or reduce the complications of bone metastases in men with hormone refractory prostate cancer is not recommended owing to insufficient evidence of efficacy. However, consider bisphosphonates for pain relief in such cases when other treatments (including analgesics and palliative radiotherapy) have failed. The oral or intravenous route of administration should be chosen according to convenience, tolerability, and cost.

- Strontium-89 should be considered for men with hormone refractory prostate cancer and painful bone metastases, especially those men who are unlikely to receive myelosuppressive chemotherapy.

- Men with hormone refractory prostate cancer shown to have extensive disease in the spine (for example, on a bone scan) should have magnetic 
resonance imaging of the spine if they develop any spinal related symptoms, as the risk of spinal cord compression is high.

\section{Treatment complications}

- For men treated with radical radiotherapy, offer follow-up with flexible sigmoidoscopy every five years as an alternative to faecal occult blood testing in the bowel screening programme.

- Healthcare professionals should ensure that men with troublesome urinary symptoms after treatment have access to specialist continence services.

- Offer early and ongoing access to specialist erectile dysfunction services to men and their partners.

\section{Follow-up}

- For men who have had stable PSA levels for at least two years after radical treatment and no significant treatment complications, offer followup outside hospital (for example, in primary care) by secure electronic communications, unless taking part in a clinical trial that requires formal, clinic based follow-up. Offer direct access to the urological cancer multidisciplinary team.

- For men with localised prostate cancer who have chosen a watchful waiting regimen with no curative intent (whereby hormonal therapy is withheld until there is significant disease progres- sion), arrange follow-up in primary care in accordance with locally agreed protocols. Measure the men's PSA levels at least once a year.

\section{Overcoming barriers}

Many of these recommendations have cost implications. A costing tool developed by NICE is available now, and an implementation pack will be available shortly. Most of the recommendations can be implemented by the multidisciplinary urological cancer teams under the supervision of the cancer networks.

Contributors: All authors contributed to reviewing the evidence and writing and editing the article.

Funding: The National Collaborating Centre for Cancer was commissioned and funded by the National Institute for Health and Clinical Excellence to write this summary.

Competing interests: MB has received consultancy fees from Roche and Pfizer and advisory fees from Speciality European Pharma, and he has attended advisory boards for Pharmion. JG has received advisory fees from Speciality European Pharma, honorariums from Sanofi-Aventis, and conference expenses from Bayer Pharmaceuticals, Sanofi-Aventis, AstraZeneca; he has also been principal investigator of trials funded by Boehringer Ingelheim, Immunicon, Cell Genesys, GPC Biotech, and SanofiAventis. FM is chief investigator of a national trial partly funded by Pfizer. The Guideline Development Group complied with NICE's policy on declarations of interests for bodies that produce guidance.

Provenance and peer review: Commissioned; not externally peer reviewed.

1 National Institute for Health and Clinical Excellence. Prostate cancer: diagnosis and treatment. London: NICE, 2008. www.nice.org.uk/CG058.

\title{
Commentary: controversies in NICE guidance on prostate cancer
}

\author{
Timothy J Wilt
}

Minneapolis VA Center for Chronic Disease Outcomes Research, 1 Veterans Drive (111-0), Minneapolis, MN 55417 Tim.wilt@med.va.gov

BMJ 2008;336:612-4 doi:10.1136/bmi.39501.621690.AD
The NICE guidelines on prostate cancer provide comprehensive advice on best practice for diagnosis and treatment of prostate cancer. They are based on systematic reviews of the evidence, incorporate multidisciplinary opinions, and try to balance the values of healthcare providers and patients for various outcomes while emphasising a patient centred approach. Their conclusions are generally consistent with other reviews and guidelines evaluating similar evidence. ${ }^{1-5}$ If followed, these recommendations will likely improve prostate cancer outcomes while reducing unnecessary, ineffective, harmful, and costly care.

\section{Paucity of randomised controlled trials}

Although NICE's recommendations are generally appropriate, any guidelines on prostate cancer are going to be hampered by the lack of high quality information available. The paucity of randomised trials limits the quality of data used for informed decision making, particularly regarding detection and treatment of localised disease. Even where randomised trials have shown benefits, the absolute magnitude of benefit is generally small, requires many years to accrue, and must be weighed against accompanying harms and costs. Few studies enrolled men with disease that had been detected as a result of measuring the level of prostate specific antigen (PSA) or were adequately powered to assess survival outcomes. Individual patients and providers may place different values on a particular outcome or have clinical and tumour characteristics that have not been adequately assessed by the trials providing the evidence used for drafting guidelines. Thus, it is difficult to mandate rigid adherence to these recommendations or to create national practice standards.

\section{Limitations that may reduce uptake by clinicians}

The full NICE guideline is wide ranging and can serve as a single source of information on prostate cancer. However, an individual clinician or patient will find that many sections are not pertinent to their situation. The broad scope makes the guideline more 